Popova, E.

\title{
Quality of the Solution Sets of Parameter-Dependent Interval Linear Systems
}

\begin{abstract}
Consider linear systems whose matrix and right-hand side vector depend affine-linearly on parameters varying within prescribed bounds We present some sufficient conditions under which the interval hull (or some bounds) of the solution set of a parametrised interval linear system coincides with the interval hull (or bounds) of the non-parametric interval linear system corresponding to the parametric one.
\end{abstract}

Keywords: interval linear systems, parameter dependence, solution set, exact bounds

MSC (1991): 65F05,65G10

\section{Introduction}

By $\mathbb{R}^{n}, \mathbb{R}^{n \times m}$ denote the set of real vectors with $n$ components, resp. the set of real $n \times m$ matrices. The set of all real compact intervals $[a]:=[\underline{a}, \bar{a}]:=\{a \in \mathbb{R} \mid \underline{a} \leq a \leq \bar{a}\}$ is denoted by $\mathbb{I} \mathbb{R}$. We assume the reader is familiar with interval arithmetic [1]. By $\mathbb{R}^{n}, \mathbb{I}^{n \times m}$ denote the sets of interval $n$-vectors, resp. $n \times m$ matrices.

Consider the linear system

$$
A(p) \cdot x=b(p),
$$

where $A(p) \in \mathbb{R}^{n \times n}$ and $b(p) \in \mathbb{R}^{n}$ depend affine-linearly on a parameter vector $p \in \mathbb{R}^{k}$. When $p$ varies within a range $[p] \in \mathbb{R}^{k}$, the set of solutions to all $A(p) \cdot x=b(p), p \in[p]$, called parametric solution set, is

$$
\Sigma^{p}:=\Sigma(A(p), b(p),[p]):=\left\{x \in \mathbb{R}^{n} \mid A(p) \cdot x=b(p) \text { for some } p \in[p]\right\} .
$$

Denote by $[A]=[\underline{A}, \bar{A}]:=A([p]) \in \mathbb{I}^{n \times n}$, resp. $[b]=[\underline{b}, \bar{b}]:=b([p]) \in \mathbb{I}^{n}$ the interval matrix, resp. the interval vector, obtained from $A(p)$, resp. $b(p)$, substituting $p$ by $[p]$ and performing the corresponding interval operations. Hence the interval linear system

$$
[A] \cdot x=[b], \quad \text { that is } A([p]) \cdot x=b([p])
$$

is a general non-parametric interval linear system corresponding to the parametric one. The solution set to the general system is $\Sigma^{g}:=\Sigma([A],[b]):=\left\{x \in \mathbb{R}^{n} \mid \exists A \in[A], \exists b \in[b]: A x=b\right\}$. In general, $\Sigma^{p} \subseteq \Sigma^{g}$.

The hull of a nonempty bounded subset $S$ of $\mathbb{R}^{n}$ is the interval vector $\square S:=[\inf S, \sup S]$. We say that the parametric solution set $\Sigma(A(p), b(p),[p])$ has the same quality as the corresponding non-parametric solution set $\Sigma(A([p]), b([p]))$, if $\square \Sigma^{p}=\square \Sigma^{g}$. We shall look for this quality of the parametric solution set at least at some of its components or some of their bounds. In case that the parametric solution set (some of its components or some of their bounds) possesses the quality of the general solution set, we can give exact finite characterization of the parametric solution set (some of its components or some of their bounds).

A square matrix $[A] \in \mathbb{R}^{n \times n}$ is called regular if all $A \in[A]$ are nonsingular. By $A \geq 0$ we denote a nonnegative $n \times m$ matrix, i.e., $a_{i j} \geq 0$ for $i=1, \ldots, n, j=1, \ldots, m$. A regular matrix $[A] \in \mathbb{I R}^{n \times n}$ is called inverse nonnegative if $A^{-1} \geq 0$ for all $A \in[A]$. We shall use the mid-point matrix $A_{m}=\frac{1}{2}(\underline{A}+\bar{A})$ of $[A] \in \mathbb{R}^{n \times n}$, the unit vector $e=(1, \ldots, 1)^{\top}$, and the unit matrix $E=(e, \ldots, e) \in \mathbb{R}^{n \times n}$. For $a \in \mathbb{R}$, define $\operatorname{sign}(a)=\{-1$ if $a<0 ; 0$ if $a=$ $0 ; 1$ if $a>0\}, \operatorname{sgn}(a)=\{1$ if $a \geq 0,-1$ otherwise $\}$ and apply these functions to real vectors componentwise. We define a reflexive and symmetric relation $\cong$ on the set $\{0,-1,1\}$ to itself by the following table.

\begin{tabular}{|c|c|c|c|}
\hline$\cong$ & -1 & 0 & 1 \\
\hline-1 & True & True & False \\
\hline 1 & False & True & True \\
\hline
\end{tabular}

\section{Results}

Following the notations, introduced by Rohn $[6]$, denote by $U(k):=\left\{u \in \mathbb{R}^{k}|| u \mid=(1, \ldots, 1)^{\top}\right\}$. For $[a]=[\underline{a}, \bar{a}] \in$ $\mathbb{I R}^{n}$ and $u \in U(n),\left\{a_{u}\right\}_{i}=\left\{\underline{a}_{i}\right.$ if $u_{i}=-1 ; \bar{a}_{i}$ if $\left.u_{i}=1\right\}, i=1, \ldots, n$. The following Theorem is well-known. 
Theorem 1. Let $A(p)$ be regular for $p \in[p]$ and let the functions $x(p)=A(p)^{-1} \cdot b(p)$ be monotone on the interval box $[p]$ with respect to each parameter $p_{i}, i=1, \ldots, k$. Then

$$
\square \Sigma^{p}=\left[\min _{u \in U(k)} A\left(p_{u}\right)^{-1} \cdot b\left(p_{u}\right), \max _{u \in U(k)} A\left(p_{u}\right)^{-1} \cdot b\left(p_{u}\right)\right] .
$$

If the monotonicity conditions are proven, then the interval hull of the parametric solution set $\Sigma^{p}$ can be found by solving at most $2^{k}$ point linear systems. However, verifying the monotonicity condition of Theorem 1 is difficult for problems of big dimensions or problems involving many parameters. Monotonicity can also be used even when the function is not monotonic in a given interval provided the behavior of the function is sufficiently well known. If the parametric solution set has the same quality as the corresponding non-parametric solution set, then we know the behavior of the parametric solution sufficiently well to get the exact bounds of the parametric solution set. In what follows we present some more general and simple sufficient conditions (not based on the monotonicity property of the parametric solution) for verifying the quality of the parametric solution set.

We begin with considering some special kinds of linear systems the interval hull of whose general solution set can be given explicit representation. For a parametrised linear system (1) and an $u \in U(k)$ we introduce the following relations

$$
\begin{array}{lll}
b\left(p_{u}\right)=\underline{b} & \text { and } & A\left(p_{u}\right)=\bar{A} \\
b\left(p_{u}\right)=\underline{b} & \text { and } & A\left(p_{u}\right)=\underline{A} .
\end{array}
$$

Since the components of $A(p)$ depend affine-linearly on $p$, the following assertion is valid: There exists $u \in U(k)$ such that $\min _{p \in[p]} A(p)=A\left(p_{-u}\right)$, resp. $\max _{p \in[p]} A(p)=A\left(p_{u}\right)$, iff $\operatorname{sign}\left(\frac{\partial A(p)}{\partial p_{m}}\right) \cong E \vee(-E)$ for all $m=1, \ldots, k$, and $u \in U(k)$ is defined by $u_{i}=\left\{\begin{array}{ll}1 & \text { if } \operatorname{sign}\left(\frac{\partial A(p)}{\partial p_{m}}\right) \cong E, \\ -1 & \text { if } \operatorname{sign}\left(\frac{\partial A(p)}{\partial p_{m}}\right) \cong-E .\end{array}\right.$ A similar assertion is valid for $b(p)$. It is obvious that if $A$ and $b$ are "dependent" ${ }^{1}$, then only one of the relations (2), (3) may hold true (see Example 2 below). If $A$ and $b$ are independent, there may exist $u, v \in U(k), u \neq v$, such that (2) holds true for $u$ and (3) holds true for $v$ (e.g. Example 1 below).

Theorem 2. Let $[A] \in \mathbb{R}^{n \times n}$ be an inverse nonnegative matrix and $[b] \in \mathbb{R}^{n}$ be such that either $\underline{b} \geq 0$, $0 \in[b]$, or $\bar{b} \leq 0$. Then we have

$\square \Sigma^{p}=\left[A\left(p_{u}\right)^{-1} \cdot b\left(p_{u}\right), A\left(p_{-u}\right)^{-1} \cdot b\left(p_{-u}\right)\right]=\square \Sigma^{g}= \begin{cases}{\left[\bar{A}^{-1} \cdot \underline{b}, \underline{A}^{-1} \cdot \bar{b}\right]} & \text { if } \underline{b} \geq 0, \exists u \in U(k) \text { satisfying (2), } \\ {\left[\underline{A}^{-1} \cdot \underline{b}, \bar{A}^{-1} \cdot \bar{b}\right]} & \text { if } \bar{b} \leq 0, \exists u \in U(k) \text { satisfying (3); }\end{cases}$

$\inf \Sigma^{p}=A\left(p_{u}\right)^{-1} \cdot b\left(p_{u}\right)=\inf \Sigma^{g}=\underline{A}^{-1} \cdot \underline{b} \quad$ if $0 \in[b], \exists u \in U(k)$ satisfying (3);

$\sup \Sigma^{p}=A\left(p_{-v}\right)^{-1} \cdot b\left(p_{-v}\right)=\sup \Sigma^{\underline{g}}=\underline{A}^{-1} \cdot \bar{b} \quad$ if $0 \in[b], \exists v \in U(k)$ satisfying (2).

Proof: Let $[A]$ be inverse nonnegative, $\underline{b} \geq 0$, and let there exist $u \in U(k)$ such that $(2)$ holds. Since the individual components of $A(p)$ and $b(p)$ depend linearly on $p$, relation (2) implies $b\left(p_{-u}\right)=\bar{b}, A\left(p_{-u}\right)=$ $\underline{A}$. According to $\left[4\right.$, Satz 2], $\square \Sigma^{g}=\left[\bar{A}^{-1} \cdot \underline{b}, \underline{A}^{-1} \cdot \bar{b}\right] \stackrel{(2)}{=}\left[A\left(p_{u}\right)^{-1} \cdot b\left(p_{u}\right), A\left(p_{-u}\right)^{-1} \cdot b\left(p_{-u}\right)\right]$. However, $A\left(p_{u}\right)^{-1} \cdot b\left(p_{u}\right), A\left(p_{-u}\right)^{-1} \cdot b\left(p_{-u}\right) \in \Sigma^{p}$, which implies $\square \Sigma^{p}=\square \Sigma^{g}$. The rest of the theorem follows analogously.

The corresponding result for the special case of symmetric M-matrix and independent right-hand side is presented in [2].

Example 1. Let $p_{1} \in[1,2], p_{2} \in[-1,0], p_{3} \in[-2,3]$, and $A(p)=\left(\begin{array}{cc}-1 & p_{1} \\ p_{1}+1 & p_{2}\end{array}\right)$.

Consider the parametrised linear system (1) with

a) $\quad b(p)=\left(p_{3}, p_{3}\right)^{\top}$;

b) $b^{\prime}(p)=\left(-p_{1}-4 p_{2},-p_{1}-4 p_{2}\right)^{\top}$.

The interval matrix $A([p])$ is inverse nonnegative and $0 \in b([p])=b^{\prime}([p])$.

a) For $u=(-1,-1,-1)^{\top}, p_{u}=(1,-1,-2)^{\top}, A\left(p_{u}\right)=\left(\begin{array}{cc}-1 & 1 \\ 2 & -1\end{array}\right)=\underline{A}, b\left(p_{u}\right)=(-2,-2)^{\top}=\underline{b}$ (that is the relation (3)) which implies $\inf \Sigma^{p}=\inf \Sigma^{g}$. Relation (2) is satisfied for $v=(1,1,-1)^{\top}$. Hence, according to Theorem $2, \sup \Sigma^{p}=A\left(p_{-v}\right)^{-1} \cdot b\left(p_{-v}\right), p_{-v}=(1,-1,3)^{\top}, A\left(p_{-v}\right)=\underline{A}, b\left(p_{-v}\right)=(3,3)^{\top}=\bar{b}$, and $\square \Sigma^{p}=$ $([-4,6],[-6,9])^{\top}$. The parametric solution set (the gray region) and the corresponding general solution set (dashed line) are presented on Figure 1.A.

\footnotetext{
${ }^{1}$ We say that $A(p)$ and $b(p), p \in \mathbb{R}^{k}$, are "independent" if there exists partitioning $p=\left(p^{\prime}, p^{\prime \prime}\right)$ with $p^{\prime}=\left(p_{1}, \ldots, p_{k_{1}}\right) \in \mathbb{R}^{k_{1}}$, $p^{\prime \prime}=\left(p_{k_{1}}, \ldots, p_{k}\right) \in \mathbb{R}^{k-k_{1}}$ such that $A(p)=A\left(p^{\prime}\right), b(p)=b\left(p^{\prime \prime}\right) . A$ and $b$ are "dependent" if they are not independent.
} 
b) Relation (2) holds for $v=(1,1)^{\top}$. Since $A(p)$ and $b^{\prime}(p)$ are dependent, only the upper bounds of $\Sigma^{p}$ have the quality of the corresponding bounds of $\Sigma^{g}$. The region, filled in black, on Figure 1.A represents the parametric solution set.

The next Theorem follows from [6, Theorems 3.2, 4.5] and generalizes a Theorem by Beeck [5, Satz 3].

Theorem 3. Let $[A] \in \mathbb{R}^{n \times n}$ be inverse stable ${ }^{2}$. Then for each $i, 1 \leq i \leq n$

$$
\begin{aligned}
& \left\{\inf \Sigma^{g}\right\}_{i}=\left\{\underline{A}^{-1} \cdot b_{-y(i)}\right\}_{i} \quad \text { if } \operatorname{sgn}\left(A_{m}^{-1} \cdot b_{-y(i)}\right)=-y(i), \\
& \left\{\sup \Sigma^{g}\right\}_{i}=\left\{\underline{A}^{-1} \cdot b_{y(i)}\right\}_{i} \quad \text { if } \operatorname{sgn}\left(A_{m}^{-1} \cdot b_{y(i)}\right)=y(i),
\end{aligned}
$$

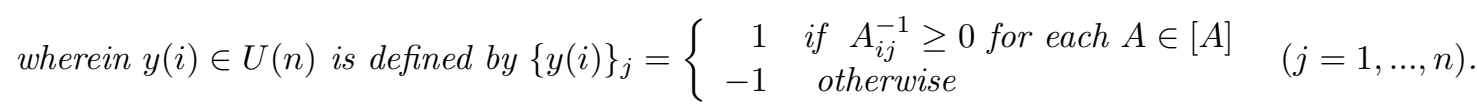

Theorem 4. Let $A([p]) \in \mathbb{R}^{n \times n}$ be inverse stable. Then for each $i, 1 \leq i \leq n$ we have

$$
\begin{aligned}
& \left\{\inf \Sigma^{p}\right\}_{i}=\left\{\inf \Sigma^{g}\right\}_{i} \quad \text { if } \operatorname{sgn}\left(A_{m}^{-1} \cdot b_{-y(i)}\right)=-y(i), \exists u \in U(k): A\left(p_{u}\right)=\underline{A}, b\left(p_{u}\right)=b_{-y(i)}, \\
& \left\{\sup \Sigma^{p}\right\}_{i}=\left\{\sup \Sigma^{g}\right\}_{i} \quad \text { if } \operatorname{sgn}\left(A_{m}^{-1} \cdot b_{y(i)}\right)=y(i), \exists v \in U(k): A\left(p_{v}\right)=\underline{A}, b\left(p_{v}\right)=b_{y(i)} .
\end{aligned}
$$

$y(i) \in U(n)$ is defined as in Theorem 3.

Proof: The proof, based on Theorem 3, is similar to the proof of Theorem 2.

Example 2. Consider the parametrised linear system (1), wherein

$$
A(p)=\left(\begin{array}{cc}
p_{1} & 2 p_{2} \\
p_{2} & p_{1}
\end{array}\right), b(p)=\left(\begin{array}{c}
\frac{1}{2} p_{1} \\
p_{3}
\end{array}\right), \quad p=\left(\begin{array}{c}
p_{1} \\
p_{2} \\
p_{3}
\end{array}\right) \in\left(\begin{array}{c}
{[2,4]} \\
{\left[\begin{array}{ll}
2 & 0
\end{array}\right]} \\
{[-4,2]}
\end{array}\right) .
$$

The interval matrix $A([p])$ is inverse nonnegative. The right-hand side does not satisfy the conditions of Theorem 2. However, we can apply Theorem 3 since $y=e$ for $i=1,2$, and $\operatorname{sgn}\left(A_{m}^{-1} \cdot \underline{b}\right)=-e, \operatorname{sgn}\left(A_{m}^{-1} \cdot \bar{b}\right)=e$. Thus we have explicit representation for the interval hull of the general solution set $\square \Sigma^{g}=\left[\underline{A}^{-1} \cdot \underline{b}, \underline{A}^{-1} \cdot \bar{b}\right]=$ $([-3,4],[-7 / 2,3])^{\top}$. Since $A\left(p_{u}\right)=\underline{A}, b\left(p_{u}\right)=\underline{b}$ for $u=(1,1,1)^{\top}$ we have according to Theorem 4 that $\inf \Sigma^{p}=\inf \Sigma^{g}=(-3,-7 / 2)^{\top}$. Due to the dependency of the matrix and the right-hand side vector, the relation (2) does not hold for any $v \in U(3)$. The solution set of the parametrised linear system (gray region) and the solution set of the corresponding general interval linear system (dashed line) are presented on Figure 1.B.

The black curve within the gray region on Fig. 1.B represents the parametric solution set to the linear system defined by the same matrix $A(p)$ and right-hand side vector $b^{\prime}(p)=\left(\frac{1}{2} p_{1}, 6 p_{2}+p_{1}\right)^{\top}$. The above considerations can be applied to this new parametric system which has the same quality as the previous one. Note that $b([p])=b^{\prime}([p])$.

In order to generalize further the above results we recall some results from [6]. For $y, z \in U(n)$ denote

$$
\left\{A_{y, z}\right\}_{i j}=\left\{\begin{array}{lll}
\underline{a}_{i j} & \text { if } & y_{i} z_{j}=1, \\
\bar{a}_{i j} & \text { if } & y_{i} z_{j}=-1,
\end{array} \quad\left\{b_{y}\right\}_{i}=\left\{\begin{array}{lll}
\bar{b}_{i} & \text { if } & y_{i}=1, \\
\underline{b}_{i} & \text { if } & y_{i}=-1 .
\end{array}\right.\right.
$$

Theorem 5. (Rohn [6], Theorem 2.2) Let $[A]$ be regular. Then for each $y \in U(n)$, the problem

$$
\begin{aligned}
A_{y, z} \cdot x & =b_{y}, \\
\operatorname{diag}(z) \cdot x & \geq 0, \quad z \in U(n)
\end{aligned}
$$

has exactly one solution $x_{y} \in \Sigma^{g}$ and $\square \Sigma^{g}=\left[\min _{y \in U(n)} x_{y}, \max _{y \in U(n)} x_{y}\right]$.

Theorem 5 implies that for each component $i=1, \ldots, n$ and each bound $j \in\{\inf , \sup \}$ of $\square \Sigma^{g}$ there exist $y \in U(n)$ and $z \in U(n)$ so that $j\left(\left\{\square \Sigma^{g}\right\}_{i}\right)=x_{y, z}$, wherein $x_{y, z}$ is a solution of problem (4).

The special kind of the interval matrix and the sign-conditions in Theorems 2-4 provide that the corresponding bound of the general solution set can be obtained by solving only one point linear system, that is the solution to the problem (4) for fixed $y, z \in U(n), y \in U(n)$ being determined by the type of the matrix and $z \in U(n)$ being determined by the corresponding sign-condition. In the general case of an arbitrary regular interval matrix $[A]$ Rohn [6] gives an iterative algorithm for computing $\square \Sigma^{g}$, based on Theorem 5. Once $\square \Sigma^{g}$ is computed, the corresponding $y(i, j), z(i, j) \in U(n), i=1, \ldots, n, j \in\{\inf , \sup \}$ are determined and we can search for the same quality of the parametric solution set at the corresponding $i, j$ as stated by the following Theorem. Denote by $y(i, j), z(i, j)$ the corresponding $y, z \in U(n)$ for which $j\left(\left\{\square \Sigma^{g}\right\}_{i}\right)=x_{y, z}$. For each $l \in\{1, \ldots, n\}, m \in\{\inf , \sup \}$ denote $T(y(l, m), z(l, m))=\left(t_{i j}\right) \in \mathbb{R}^{n \times n}, t_{i j}=y(l, m)_{i} \cdot z(l, m)_{j}, i, j=1, \ldots, n$.

\footnotetext{
${ }^{2}[A]$ is inverse stable if for each $i, j \in\{1, \ldots, n\}$, either $A_{i j}^{-1} \leq 0$ for each $A \in[A]$, or $A_{i j}^{-1} \geq 0$ for each $A \in[A]$.
} 
Theorem 6. Let $[A]$ be regular and $j\left(\left\{\square \Sigma^{g}\right\}_{i}\right)=x_{y(i, j), z(i, j)}, i=1, \ldots, n, j \in\{$ inf, sup $\}$. If there exists $u \in U(k)$ such that $A\left(p_{u}\right)=A_{y(i, j), z(i, j)}$ and $b\left(p_{u}\right)=b_{y(i, j)}$ for some $i \in\{1, \ldots, n\}$ and some $j \in\{\inf$, sup $\}$, then $j\left(\{\square \Sigma(A(p), b(p),[p])\}_{i}\right)=j\left(\{\square \Sigma([A],[b])\}_{i}\right)$.

Proof: The proof follows from $A\left(p_{u}\right)^{-1} b\left(p_{u}\right) \in \Sigma^{p}, A\left(p_{u}\right)^{-1} b\left(p_{u}\right) \in \square \Sigma^{g}$ and $\square \Sigma^{p} \subseteq \square \Sigma^{g}$.

Example 3. Consider the parametrised linear system (1), wherein

$$
A(p)=\left(\begin{array}{cc}
p_{1} & 2 p_{2} \\
p_{2} & p_{1}
\end{array}\right), b(p)=\left(\begin{array}{c}
p_{3} \\
p_{4}
\end{array}\right), \quad p \in([2,4],[-1,0],[17 / 3,7],[-17,-1])^{\top} .
$$

The interval matrix $A([p])$ is inverse nonnegative. Hence, in Theorem $3, y(1)=y(2)=-e$ for the inf-components, $y(1)=y(2)=e$ for the sup-components and we find $A_{m}^{-1} \underline{b} \neq-e, A_{m}^{-1} \bar{b}=e$. Hence by Theorem 3 we can find explicit representation only for the upper bounds of the general solution set. Since $A\left(p_{v}\right)=\underline{A}$ and $b\left(p_{v}\right)=$ $\bar{b}$ for $v=(-1,-1,1,1)^{\top}$, we can apply Theorem 4 to get the exact upper bounds of the parametric solution set $\sup \Sigma^{p}=(6,5 / 2)^{\top}$. Applying the iterative Rohn's algorithm for computing $\square \Sigma^{g}$ we find that both lower bounds of the general solution set are attained for $y(i, 1)=(-1,-1)^{\top}$ and $z(i, 1)=(-1,-1)^{\top}, i=1,2$. Since $A\left(p_{u}\right)=A_{y(i, 1), z(i, 1)}=\underline{A}, b\left(p_{u}\right)=b_{y(i, 1)}=\underline{b}, i=1,2$ for $u=(-1,-1,-1,-1)^{\top}$, by Theorem 6 we find that the parametric solution set has the same quality as the corresponding general solution set at the lower bounds, too. Thus $\square \Sigma^{p}=([-34 / 3,6],[-86 / 6,5 / 2])^{\top}$.

So far, verifying the quality of the parametric solution set for fixed $y, z \in U(n)$, it was necessary to test the relation $A\left(p_{u}\right)=A_{y, z} \vee b\left(p_{u}\right)=b_{y}$ for all $2^{k} u \in U(k)$. The next Theorem reduces the number of the verified relations to $k$.

Theorem 7. Let $A(p) \cdot x=b(p), p \in[p] \in \mathbb{R}^{n}$ be a parametrised linear system. Let the interval matrix $A([p])$ is regular and $j\left(\left\{\square \Sigma^{g}\right\}_{i}\right)=x_{y(i, j), z(i, j)}$ for $i=1, \ldots, n, j \in\{\inf , \sup \}, x_{y(i, j), z(i, j)}$ being the solution of (4) for particular $y=y(i, j), z=z(i, j) \in U(n)$. Then for fixed $i=1, \ldots, n, j \in\{\inf , \sup \}$ and $y=y(i, j), z=z(i, j) \in U(n)$ the following two conditions are equivalent:

I. There exists $u \in U(k)$ such that $A\left(p_{u}\right)=A_{y, z}$ and $b\left(p_{u}\right)=b_{y}$.

II. For each fixed $m=1, \ldots, k$

$$
\operatorname{sign}\left(\frac{\partial A(p)}{\partial p_{m}}\right) \cong\left\{\begin{array}{cl}
T(y, z) \vee-T(y, z) & \text { if } \operatorname{sign}\left(\frac{\partial b(p)}{\partial p_{m}}\right)=(0, \ldots, 0)^{\top}, \\
-T(y, z) & \text { if } \operatorname{sign}\left(\frac{\partial b(p)}{\partial p_{m}}\right) \cong y, \\
T(y, z) & \text { if } \operatorname{sign}\left(\frac{\partial b(p)}{\partial p_{m}}\right) \cong-y .
\end{array}\right.
$$

Proof: Let (I.) hold. Using the notation $a^{\lambda}=\{\underline{a}$ if $\lambda=-1 ; \bar{a}$ if $\lambda=1\}$ for $[a]=[\underline{a}, \bar{a}] \in \mathbb{R}$ and $\lambda \in\{1,-1\}$, we have for $i, j=1, \ldots, n\left\{A_{y, z}\right\}_{i j}=\{A\}_{i j}^{\lambda_{i j}}$ and $\left\{b_{y}\right\}_{i}=\{b\}_{i}^{\mu_{i}}$ wherein $\lambda_{i j}=-y_{i} z_{j}, \mu_{i}=y_{i}$, that is $\left\{a\left(p_{u}\right)\right\}_{i j}=a_{i j}^{-y_{i} z_{j}}$ and $\left\{b\left(p_{u}\right)\right\}_{i}=b_{i}^{y_{i}}$ for all $i, j=1, \ldots, n$. Since all components of $A(p)$ and $b(p)$ depend affine-linearly on $p_{m}, m=1, \ldots, k$,

$$
\left\{a\left(p_{u}\right)\right\}_{i j}=\alpha_{i j 1} p_{1}^{u_{1}}+\ldots+\alpha_{i j k} p_{k}^{u_{k}}, \quad\left\{b\left(p_{u}\right)\right\}_{i}=\beta_{i 1} p_{1}^{u_{1}}+\ldots+\beta_{i k} p_{k}^{u_{k}}, \quad i, j=1, \ldots, n,
$$

the corresponding inf/sup bounds of $[a]_{i j},[b]_{i}$ will be attained at the corresponding inf/sup bounds of the additive intervals $\alpha_{m}[p]_{m}, \beta_{m}[p]_{m}$. Hence (I.) is equivalent to $\operatorname{sign}\left(\alpha_{i j m}\right) u_{m} \cong-y_{i} z_{j}$ and $\operatorname{sign}\left(\beta_{i m}\right) u_{m} \cong y_{i}$ for all $i, j=$ $1, \ldots, n$ and $m=1, \ldots, k$, which implies that (I.) is equivalent to $u_{m} \cdot \operatorname{sign}\left(\frac{\partial b(p)}{\partial p_{m}}\right) \cong y$ and $u_{m} \cdot \operatorname{sign}\left(\frac{\partial A(p)}{\partial p_{m}}\right) \cong$ $-T(y, z)$ for each fixed $m \in\{1, \ldots, k\}$, which leads to (II.).

Let (II.) hold. Defining

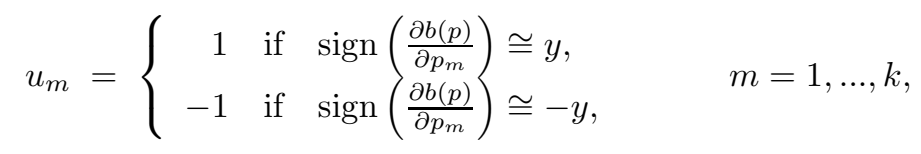

we obtain $A\left(p_{u}\right)=A_{y, z}$ and $b\left(p_{u}\right)=b_{y}$.

Using Theorem 7 we can reformulate Theorem 2, Theorem 4 and Theorem 6 in terms of the sign-condition II. In particular, for an inverse nonnegative interval matrix $\square \Sigma^{g}=\left[x_{-e}, x_{e}\right]$, where $x_{-e}, x_{e}$ are solutions to (4); the relation (2) is equivalent to $z=-y$, the relation (3) is equivalent to $z=y$.

To implement Theorem 6 we can extend Rohn's algorithm [6] for computing $x_{y}$-s by a verifying procedure based on the sign condition II. However, Rohn's algorithm is designed to minimize the number of the end-point linear systems solved. It may happen, as the following example shows, that at some components the corresponding inf / sup bound of $\Sigma^{g}$ is determined by more than one end-point linear systems. One of these systems could satisfy the parametric condition but might not be a vertex of $\Sigma^{g}$, determined by the Rohn's algorithm. 
Example 4. ([3]) Consider the parametrised linear system ${ }^{3}$ (1), wherein

$$
A(p)=\left(\begin{array}{cc}
1 & p_{1} \\
p_{1} & p_{2}
\end{array}\right), b(p)=\left(\begin{array}{c}
p_{3} \\
p_{3}
\end{array}\right), \quad p=\left(\begin{array}{c}
p_{1} \\
p_{2} \\
p_{3}
\end{array}\right) \in\left(\begin{array}{c}
{[0,1]} \\
{[-4,-1]} \\
{[0,2]}
\end{array}\right)
$$

As seen on Figure 1.C, representing the parametric and the general solution sets, $\inf \Sigma^{p}=\inf \Sigma^{g}$. Applying Theorem 6 we obtain that $\left\{\inf \Sigma^{g}\right\}_{1}$ is attained for $y=(-1,-1)^{\top}=-z$ and the corresponding conditions (II.) hold. Since $\left\{\inf \Sigma^{g}\right\}_{2}$ is attained for $y=(-1,1)^{\top}=-z$ but $\operatorname{sign}\left(\frac{\partial b(p)}{\partial p_{3}}\right) \varsubsetneqq y$, Theorem 6 cannot be applied to $\left\{\inf \Sigma^{p}\right\}_{2}$. Five different end-point systems have solutions (the solid points on Fig.1C) whose second component is $x_{2}=-2=\inf \Sigma_{2}^{g}$. One of these systems, $\tilde{A} \cdot x=\tilde{b}$ with $x_{2}=-2$, is such that there exists $u=(-1,1,1)^{\top}$ with the property $A\left(p_{u}\right)=\tilde{A}, b\left(p_{u}\right)=\tilde{b}$, which proves that $\left\{\inf \Sigma^{p}\right\}_{2}=\left\{\inf \Sigma^{g}\right\}_{2}$ for this example.
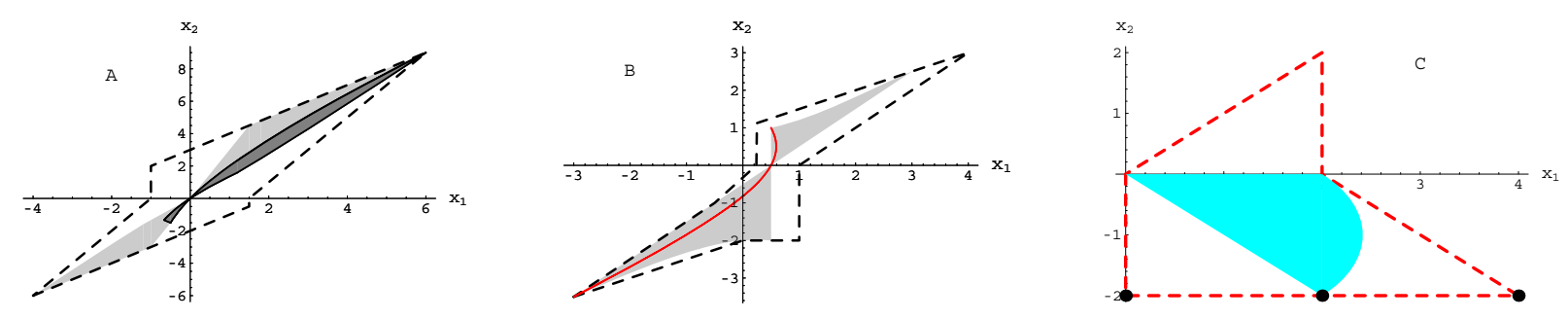

Figure 1: The parametrised (filled in region) and the general (dashed line) solution sets for the linear systems of Example 1 (A), Example 2 (B) and Example 4 (C).

For completeness, we give the following Theorem:

Theorem 8. Let $A([p])$ be regular and let for fixed $i \in\{1, \ldots, n\}, j \in\{\mathrm{inf}, \sup \}$

$$
\mathcal{S}(i, j):=\left\{\{\tilde{A}, \tilde{b}\} \mid j\left(\square \Sigma_{i}^{g}\right)=\tilde{A}^{-1} \tilde{b} \text { and } \tilde{a}_{k l} \in\left\{\underline{a}_{k l}, \bar{a}_{k l}\right\}, \tilde{b}_{k} \in\left\{\underline{b}_{k}, \bar{b}_{k}\right\} ; k, l=1, \ldots, n\right\}
$$

Then $j\left(\square \Sigma_{i}^{p}\right)=j\left(\square \Sigma_{i}^{g}\right)$ iff there exists $t \in U(k)$ such that $A\left(p_{t}\right)=\tilde{A}$ and $b\left(p_{t}\right)=\tilde{b}$ for some $\{\tilde{A}, \tilde{b}\} \in \mathcal{S}(i, j)$.

Theorems 2, 4 and 6 define sufficient conditions for comparing $\square \Sigma^{p}$ to $\square \Sigma^{g}$ and require considerably less computational effort than a general procedure, based on Theorem 8. The general procedure and the procedure, based on Theorem 6, are implemented in a Mathematica [7] package.

Acknowledgement. This work was supported by the Bulgarian National Science Fund grant No. I-903/99. The author thanks to the anonymous referees for their valuable remarks which improved the quality of the paper.

\section{References}

1 Alefeld, G.; Herzberger, J.: Introduction to Interval Computations. Academic Press, New York 1983.

2 Alefeld, G.; Mayer, G.: The Cholesky Method for Interval Data. LAA 194 (1993), pp. 161 - 182.

3 Alefeld, G.; Kreinovich, V.; Mayer, G.: On the Shape of the Symmetric, Persymmetric, and Skew-Symmetric Solution Set. SIAM J. Matrix Anal. Appl. 18, 3 (1997), pp. 693 - 705.

4 BEeck, H.: Zur scharfen Aussenabschätzung der Lösungsmenge bei linearen Intervallgleichungssystemen. ZAMM 54 (1974), T 208 - T 209.

5 Beeck, H.: Zur Problematik der Hüllenbestimmung von Intervallgleichungssystemen. In: NickeL, K. (ed.): Interval Mathematics. Lecture Notes in Computer Science 29, Springer-Verlag, 1975, pp. 150 - 159.

6 Rohn, J.: Systems of Linear Interval Equations. Linear Algebra and its Applications 126 (1989), pp. 39 -78.

7 Wolfram, S.: The Mathematica Book, 4th ed., Wolfram Media/Cambridge University Press, 1999.

Received February 21, 2001, revised October 15, 2001, accepted November 22, 2001

Address: Dr. Evgeniua D. Popova, Institute of Mathematics \& Informatics, Bulgarian Academy of Sciences, Acad. G. Bonchev Str., block 8, BG-1113 Sofia, Bulgaria, email: epopova@iph.bio.bas.bg

\footnotetext{
${ }^{3}$ The corresponding linear system with symmetric matrix and independent components of the right-hand side is considered in [3].
} 